\title{
A Method of Force Output Test for a Low Thrust High Precision Flat Space Electromagnetism Actuator
}

\author{
Chengchen Deng ${ }^{1}$, Zhongxing Tang ${ }^{1}{ }^{*}$, Min Zhu ${ }^{1}$, Hongbo Zhao ${ }^{1}$, He Liao ${ }^{1}$ \\ ${ }^{1}$ Shanghai Institute of Satellite Engineering, Shanghai, 200240, China \\ ayujishenlan@163.com
}

\begin{abstract}
Keywords: Flat space electromagnetism actuator, Low thrust, High precision, Micro vibration suppress.
\end{abstract}

\begin{abstract}
Future satellites require higher and higher payload's pointing precision and attitude stability, it is urgent to suppress micro vibration efficiently. This article designs a low thrust, high precision flat electromagnetism actuator. Firstly, the article designs the configuration of the actuator .Secondly, based on the working principle this article analysis data stimulation result of output force using the software-Ansoft Maxwell. Thirdly, aiming at testing the extremely low force output of an actuator, this article designs a new method based on single axe airing bear and high precision gyro. The simulation and examination show validity of the micro vibration suppression through the configuration and the force testing method.
\end{abstract}

\section{Introduction}

Recently, as the development of the science and technology, there are lots of application field in the future civil and commercial aviation. In order to settle for the high resolvability and orientation requirement, higher attitude pointing precision and stability is required for the satellite platform. Most research indicated that the micro vibration of the optics loads which frequency between 1 to $200 \mathrm{~Hz}$ must be suppressed to $0.1 \sim 1 \mathrm{mg}$ or much lower. For example, the micro vibration must be depressed to under $1 \mu \mathrm{g}$ while those frequency between 0.01 to $300 \mathrm{~Hz}$ for the scientific experimentation in the International Space Station [1-3]. It desiderates to explore the vibration isolation technique to satisfy the future observation mission.

There are about four kind of measures to suppress the vibration, which are passive, active, mixing and semi-active vibration isolations. The passive vibration isolation is a compact linker that doesn't need power and information. It's simple and dependable. It could suppress the high frequency vibration but useless for the low frequency. When the vibration frequency between $\sqrt{2} \omega_{n} / 2$ and $\sqrt{2} \omega_{\mathrm{n}}$ the vibration would be enlarged. The active vibration isolation could overcome the disadvantage of the passive vibration isolation. It could provide an intelligent and quick respond when the configuration change as time going. But the disadvantage of the active vibration isolation is that it needs much more energy and more complicated control law and may lead to instable system. The VISS and SUITE vibration isolation system that develop by Air Army Research is mixed the passive damping and active voice coil actuator. They are used to suppress the vibration which frequency between 5 to $250 \mathrm{~Hz}$ [4-6]. Thus, the voice coil actuator is a much important part of the vibration isolation system. In this article, a low thrust high precision space electromagnetism actuator is designed. Then the thrust stimulation of the space electromagnetism actuator is done to validate its output force precision. Finally, due to the much low thrust of the space electromagnetism actuator, a low thrust testing method is deployed.

\section{Working Principle of Flat Space Electromagnetism Actuator}

It is closely linked about the high precision thrust control and high bandwidth response and the form of the space electromagnetism actuator. There are mainly two kinds of configuration of the space electromagnetism actuator, which are cylinder and flat. It is difficult to machining the cylinder 
configuration of the space electromagnetism actuator. Besides, the radiating of the loop winding is difficult, too. Meanwhile, it's easy to be collided when the cylinder space electromagnetism actuator working on the vibration condition. Unlike the cylinder configuration of the space electromagnetism actuator, the flat space electromagnetism actuator has more advantage in the high precision about the machining, the traces of the printed board and the output force. Thus, we design a flat configuration space electromagnetism actuator, which has better uniformity of the magnetic field and high precision of the output force.

The working principle of the flat space electromagnetism actuator is shown in Fig. 1. The permanent magnets are fixing on the magnet support. The magnetic field which the permanent magnets engender is passed through the winding support hold in the based platform. The winding support is made up of two loop windings. One is coiled in the horizon and the other is coiled in the vertical way. When these two loop windings is transmitted electric current, they will engender the Lorenz force in the vertical and horizon. The Lorenz force will drive the magnet support to move in the vertical and horizon. As showed in Fig. 2.



Fig. 1 Working principle of the flat space electromagnetism actuator.

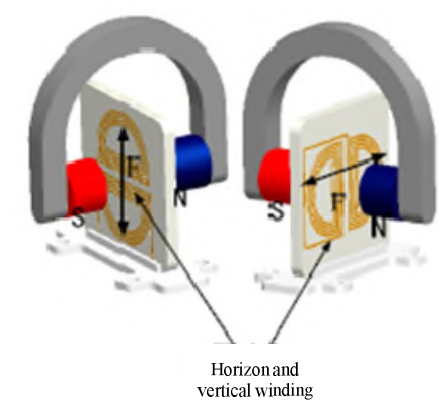

Fig. 2 Lorenz force of the flat space electromagnetism actuator.

The Lorenz force of the flat space electromagnetism actuator is correlative to the magnetic flux density $\mathrm{B}$, the length of the loop winding $\mathrm{L}$, and the input electric current $\mathrm{I}$. The equation of the Lorenz force $\mathrm{F}$ is shown as follow.

$$
F=B L I
$$

The item BL is the designed parameters of the flat space electromagnetism actuator. When the actuator is input the current $\mathrm{I}$, it will generate the Lorenz force $\mathrm{F}$ to shift the distance between the magnet support and the winding support. Due to the osculant characteristic of the flat space electromagnetism actuator, it can be adopted to suppress the micro vibration to control the attitude of the satellite for high precision observation. 
While the permanent magnets local in the balance position of the loop winding, input the current to the horizon and vertical winding and the direction of the Lorenz force is shown in Fig. 3. The yellow arrows are the current direction of horizon winding while the red arrows are the current direction of vertical. The direction of magnetic field is perpendicular to the paper towards inside.

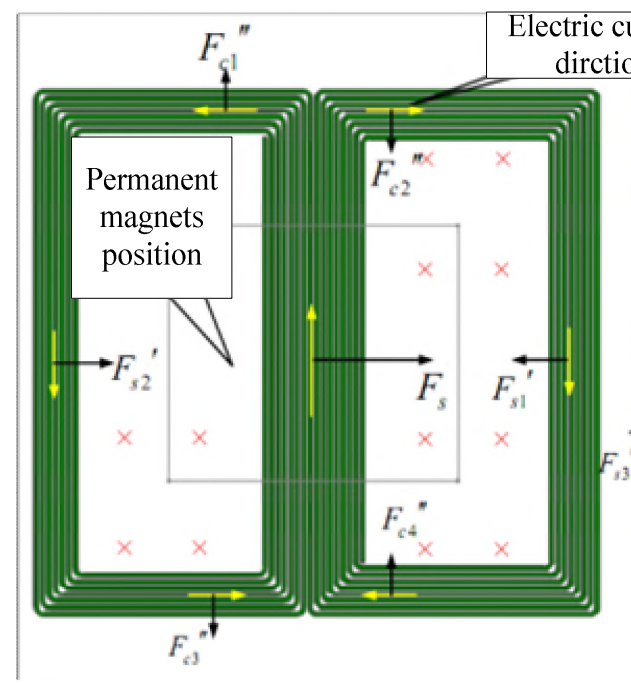

A. Horizon

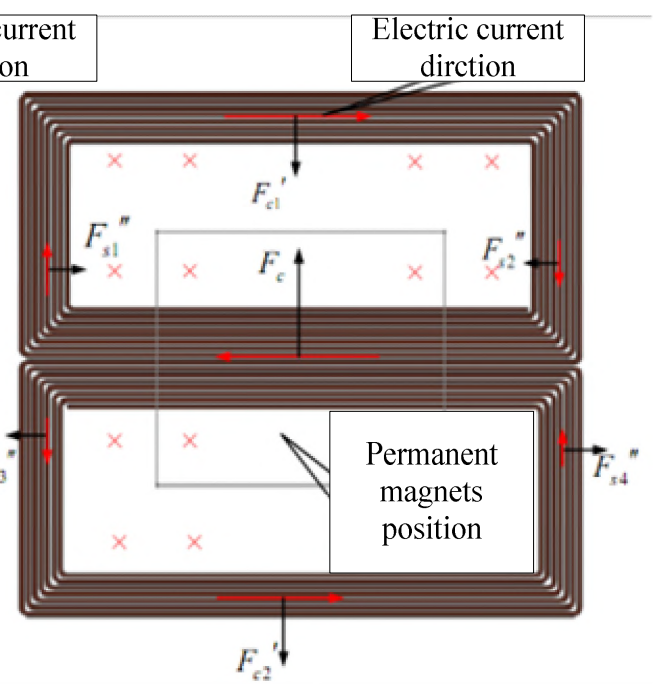

B. vertical

Fig. 3 Lorenz force of the flat space electromagnetism actuator in balance position.

While the permanent magnets local in the limit position of the loop winding, input the current to the horizon and vertical winding and the direction of the Lorenz force is shown in Fig. 4. Likely, the yellow arrows are the current direction of horizon winding while the red arrows are the current direction of vertical. The direction of magnetic field is perpendicular to the paper towards inside.

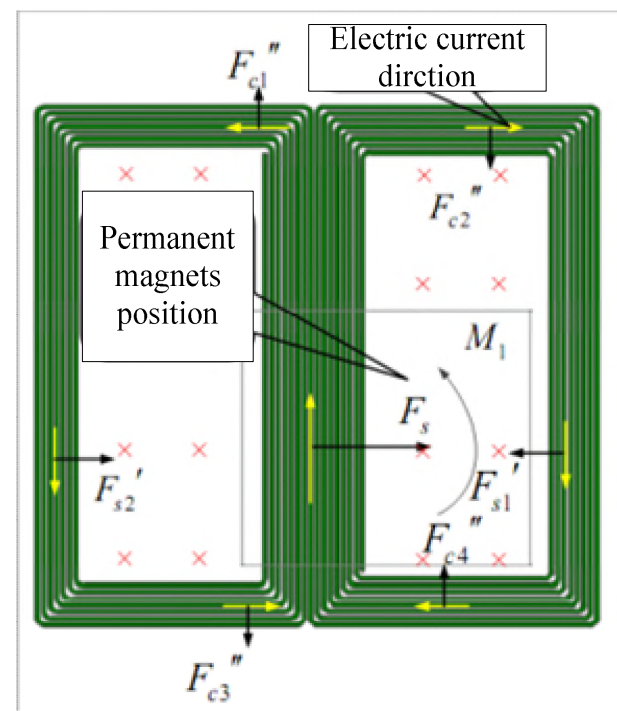

A. Horizon

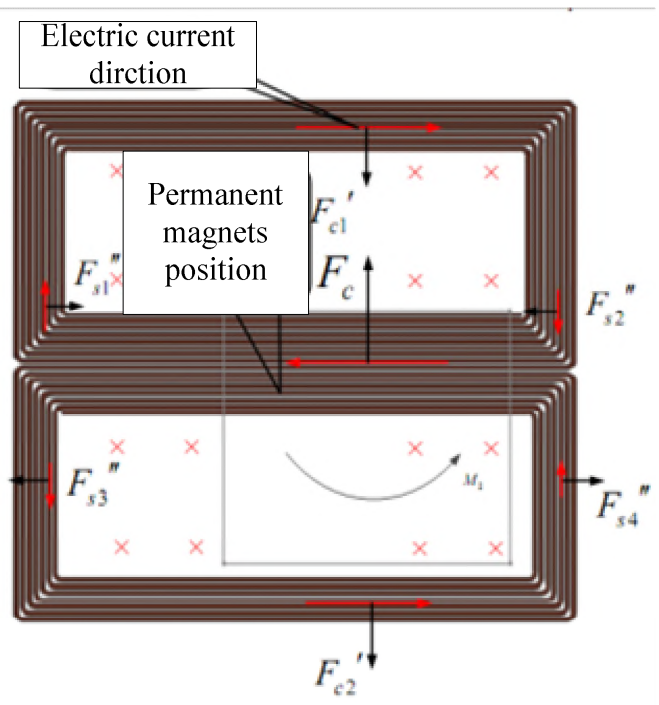

B. Vertical

Fig. 4 Lorenz force of the flat space electromagnetism actuator in limit position.

Fig. 5 is shown the position of the permanent magnets and the loop windings when the horizon and vertical superposition together. Based on the center of the loop windings, the coordinate system is constructed to describe the move between of the permanent magnets and the loop windings. The position coordinate is $(0,0) \mathrm{mm}$. 


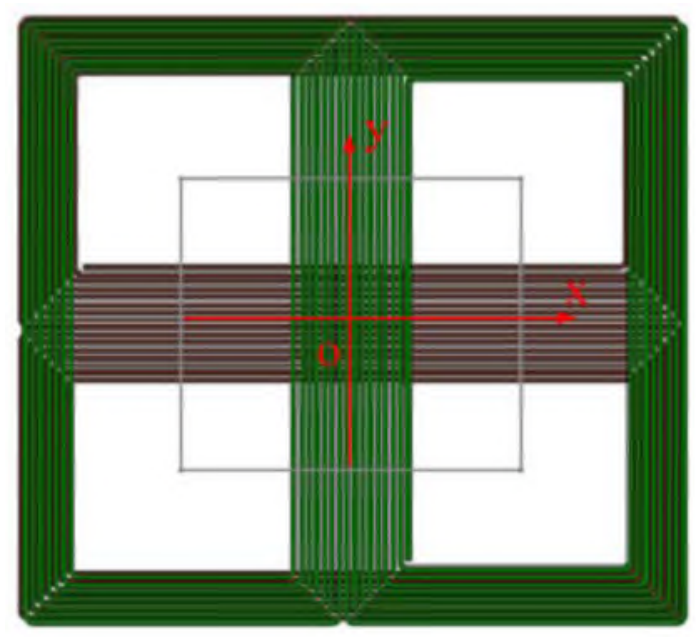

Fig. 5 Coordinate system.

The Principle sample of the flat space electromagnetism actuator is given in Fig. 6.
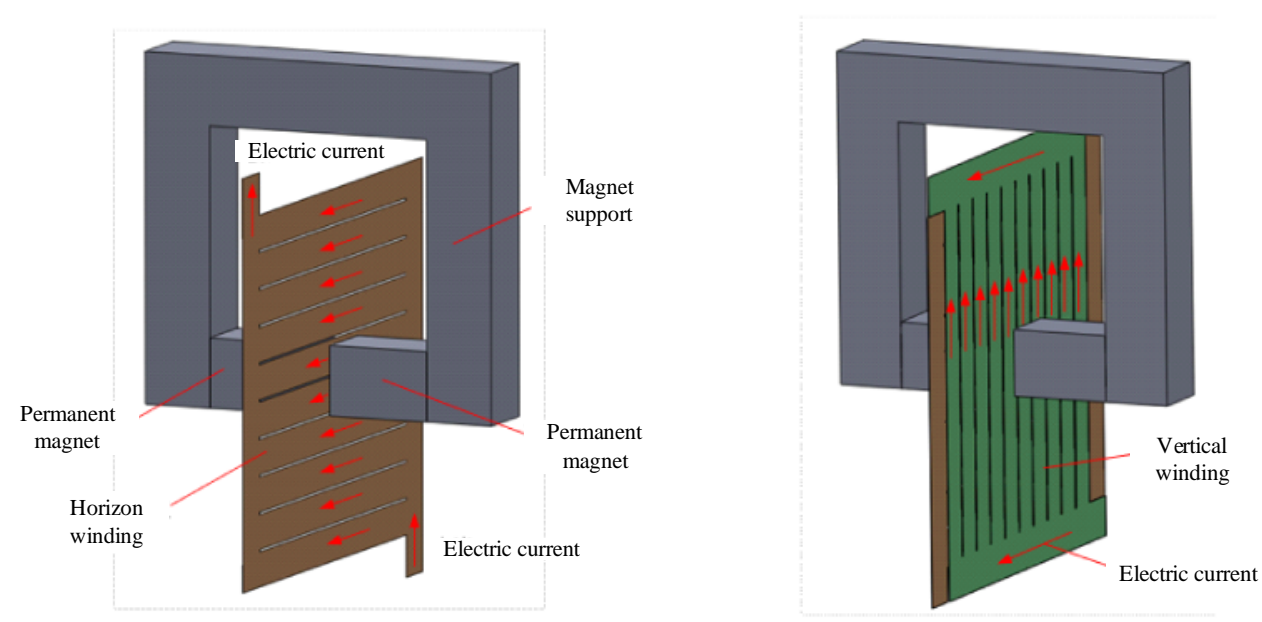

Fig. 6 Principle sample of the flat space electromagnetism actuator.

\section{Simulation of the Output Force of the Flat Space Electromagnetism Actuator}

For x moves:

The bound of the loop winding could move is $10 \mathrm{~mm}$ from left to right. We simulate relation between the distance and the output force of the flat space electromagnetism actuator. The simulation results are shown in Fig. 7 and Table 1.

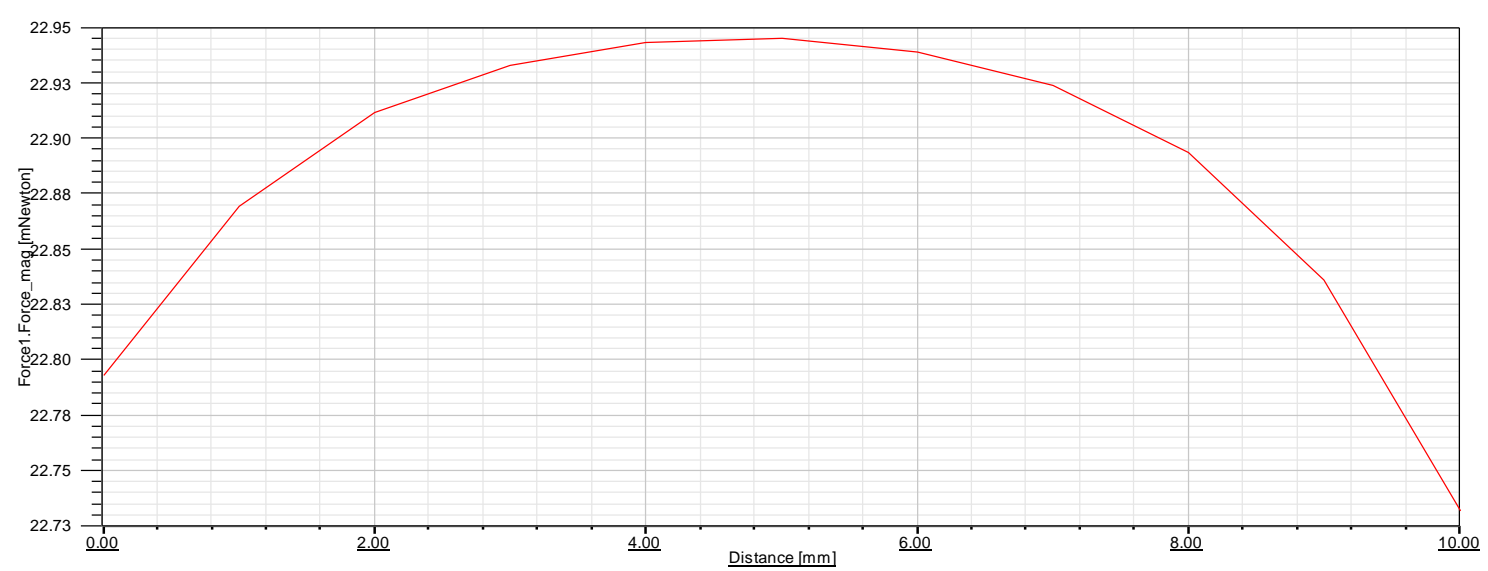

Fig. 7 Distance-force_x simulation results. 
Table 1. Distance-force_x simulation results.

\begin{tabular}{|c|c|}
\hline Distance $/ \mathrm{mm}$ & Force_x /N \\
\hline 0.000000000000000 & 54.060388836447558 \\
\hline 1.000000000000000 & 54.248506611317453 \\
\hline 2.000000000000000 & 54.350931574403972 \\
\hline 3.000000000000000 & 54.405596402876206 \\
\hline 4.000000000000000 & 54.424734809941896 \\
\hline 5.000000000000000 & 54.436272923878917 \\
\hline 6.000000000000000 & 54.424160015117707 \\
\hline 7.000000000000000 & 54.389778951880913 \\
\hline 8.000000000000000 & 54.321181191555013 \\
\hline 10.000000000000000 & 54.189920020278890 \\
\hline
\end{tabular}

For y moves:

The bound of the loop winding could move is $1 \mathrm{~mm}$ from up to down. We simulate relation between the distance and the output force of the flat space electromagnetism actuator. The simulation results are shown in Fig. 8 and Table 2.

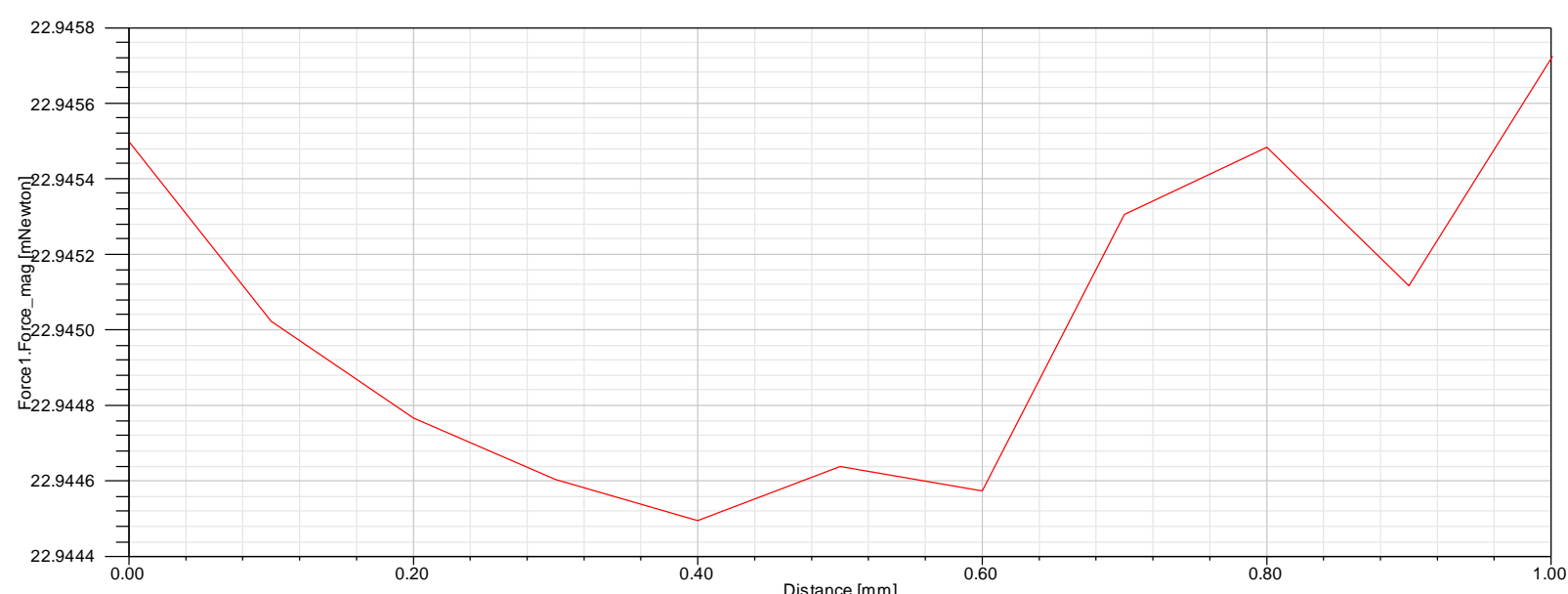

Fig. 8 Distance-force_y simulation results.

Table 2. Distance-force_y simulation results.

\begin{tabular}{|c|c|}
\hline Distance/mm & Force_y $/ \mathrm{N}$ \\
\hline 0.000000000000000 & 54.797312744987046 \\
\hline 0.100000000000000 & 54.807926223639839 \\
\hline 0.200000000000000 & 54.837039071815319 \\
\hline 0.300000000000000 & 54.821664124403028 \\
\hline 0.400000000000000 & 54.828765898740308 \\
\hline 0.500000000000000 & 54.781732418663104 \\
\hline 0.600000000000000 & 54.779226792960991 \\
\hline 0.700000000000000 & 54.781038065908582 \\
\hline 0.800000000000000 & 54.813706945605460 \\
\hline 0.900000000000000 & 54.805524260498345 \\
\hline 1.000000000000000 & 54.797312744987046 \\
\hline
\end{tabular}

From the simulation results as Fig. 7, 8 and Table 1, 2, it could be seen that the uniformity of the output force is good. The error of the output force of the flat space electromagnetism actuator is less $5 \times 10^{-5} \mathrm{~N}$. 
However, it's very difficult to test so low thrust force of the high precision flat space electromagnetism actuator output as there is no ergometer has so high resolving ablity. Thus, we develop a method to test such low thrust force of the high precision flat space electromagnetism actuator output in the next section.

\section{Method of the Output Force Test of the High Precision Flat Space Electromagnetism Actuator}

Since the output force of the high precision flat space electromagnetism actuator is too small, it's unable to directly test the amplitude of the output force of the high precision flat space electromagnetism actuator. So, we transfer the output force test to the running test of the object that impulses by the force product by the high precision flat space electromagnetism actuator. The object is designed as a cabin support by the air bearing platform that the cabin could running by very small impulse affect. From above, we can get that the high precision flat space electromagnetism actuator is working through the Lorenz effect. We hypotheses that the actuator is well working by Lorenz effect without other influence. Then the moment of the high precision flat space electromagnetism actuator brings could be calculate as follow equation.

\section{$T=F \times L_{1}$}

In Eq. 2, $\mathrm{F}(\mathrm{N})$ and $\mathrm{L}_{1}(\mathrm{~m})$ denote the output force of the high precision flat space electromagnetism actuator and the arm of it separately. T(N.m) denotes the moment of the high precision flat space electromagnetism actuator generates.

The movement of the cabin with the processing of the moment of the high precision flat space electromagnetism actuator generates could be wrote as Eq. 3.

$$
T=J \frac{d \omega}{d t}
$$

In Eq. 3, $\omega(\mathrm{rad} / \mathrm{s})$ denotes the angular velocity of the cabin. And $J\left(16.8 \mathrm{~kg} \bullet \mathrm{m}^{2}\right)$ represents the moment of inertia of the cabin, which could be gained by high precision fly wheel and gyroscope and its accuracy could reach $10^{-4} \mathrm{kgm}^{2}$ magnitude. Thus it can be seen, the output force of the high precision flat space electromagnetism actuator could be formulated as follow.

$$
F=J \frac{d \omega}{d t} / L_{1}
$$

As shown in Fig. 9, the permanent magnets support part of the high precision flat space electromagnetism actuator is setting on the wall. And the loop winding part is setting on the face A of the cabin. The distance from the center of the face $A$ to the center of the winding is $\mathrm{L}_{1}$. At the beginning of the output force test, the face A of the cabin is parallel to the wall while the high precision flat space electromagnetism actuator working well.

The angular velocity of the cabin is measured by a high precision gyroscope which fixing on the cabin. The precision of the gyroscope could be $10^{-5} \mathrm{deg} / \mathrm{s}$ and the time cost of the test is about 4 second. The angular acceleration of the cabin could be gained through difference operation, which accuracy could reach $2 \times 10^{-5} \mathrm{deg} / \mathrm{s}^{2}\left(3.5 \times 10^{-7} \mathrm{rad} / \mathrm{s}^{2}\right)$ to satisfy the request of the small force test. The distance $\mathrm{L}_{1}$ is a constant and could be measure by laser ranging and its accuracy could reach $0.05 \mathrm{~mm}$. Then all the data of the angular velocity of the cabin is recorded by the computer on the cabin. Using this method, we could gain the small output force of the high precision flat space electromagnetism actuator. The precision of the output force gets by this method could be $2 \times 10^{-5} \mathrm{~N}$. 


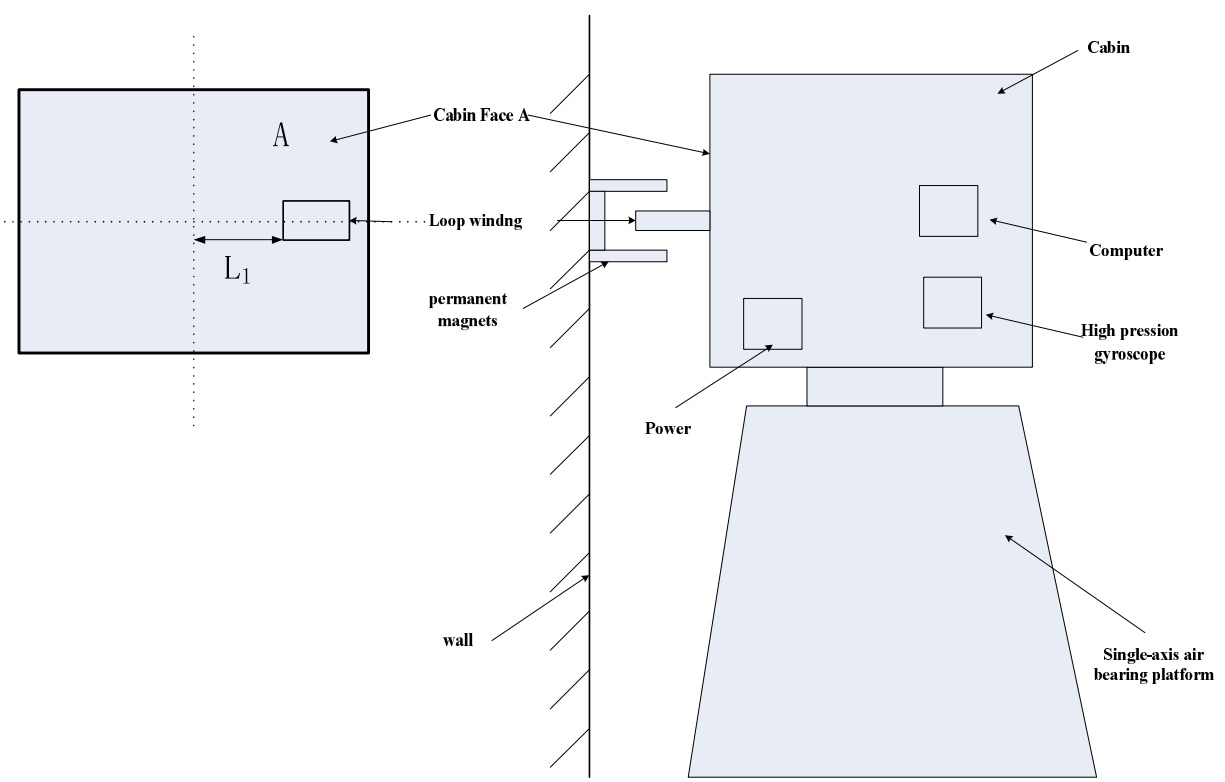

Fig. 9 Method of the output force test.

\section{Results Analysis of the Output Force Test}

In the output force test, we gave several electric currents to the high precision flat space electromagnetism actuator. The minimum output force of the electromagnetism actuator we test is about $10^{-3} \mathrm{~N}$. Then the cabin turn under the effect of the output force of the high precision flat space electromagnetism actuator. At the same time, the computer records the data of the angular velocity of the cabin. In order to reduce the effect of the air sticky, the input currents are a little larger which is still in the permissible range. The test results are shown in Table 3 and Fig. 10 is shown the angular velocity and the angular acceleration of the cabin while time going.

Table 3. Cabin turning test results.

\begin{tabular}{|c|c|c|}
\hline $\mathrm{I} /(\mathrm{A})$ & $\sigma(\mathrm{d} \omega / \mathrm{dt}))$ & $\sigma(\mathrm{F})$ \\
\hline 0.06 & $1.6462 \mathrm{e}-04$ & 0.0072 \\
\hline 0.07 & $2.0497 \mathrm{e}-04$ & 0.0090 \\
\hline 0.08 & $2.0219 \mathrm{e}-04$ & 0.0089 \\
\hline 0.09 & $2.2034 \mathrm{e}-04$ & 0.0097 \\
\hline 0.10 & $2.3774 \mathrm{e}-04$ & 0.0104 \\
\hline
\end{tabular}


Fig. 10 Result of the output force test.

From the results of the output force of the high precision flat space electromagnetism actuator presented above, we can see that the error of the output force of the flat space electromagnetism actuator is more about $7 \times 10^{-3} \mathrm{~N}$, which means the results of the test is consistent with the principle sample we used. If the output force of the electromagnetism actuator is designed limit to $1 \times 10^{-5} \mathrm{~N}$, we 
could adopt the method above to gain the output force precision. The results of the output force of the high precision flat space electromagnetism actuator is verified the feasibility of the method that transferring the output force test to the running test of the object that impulses by the force product by the high precision flat space electromagnetism actuator based on the single-axis air bearing platform and the high precision gyro.

\section{Conclusion}

A low thrust high precision flat space electromagnetism actuator is designed in this paper. The simulation result shows that this electromagnetism actuator could output a low thrust while its precision is high, that the error of the output force of the flat space electromagnetism actuator is less $5 \times 10^{-5} \mathrm{~N}$. Based on the single-axis air bearing platform and the high precision gyro, a method that transferring the much small output force test to the running test of the object that impulses by the force product by the high precision flat space electromagnetism actuator has been introduced. The results of the output force test validate the availability of the method.

\section{References}

[1] M. Miyaji, M. Yura and M. Iwasaki, Improvement of tracking accuracy of double-column machining center with high-speed and high-acceleration driving. IEEE T. Electr. Electron. Eng. 4(2) (2009) 210-217.

[2] Q. Hu, H. Li and H. Yu, et al. Neural network sliding mode controller for linear elevator using permanent magnet linear synchronous motor. 5th International Conference on Natural Computation, ICNC 2009, Tianjian, China, 2009.

[3] E. B. Pilinski and A. Y. Lee, Pointing-Stability Performance of the Cassini Spacecraft. J. Spacecraft. Rockets. 46(5) (2009) 1007-1015.

[4] L. Dewell, Precision Telescope Pointing and Spacecraft Vibration Isolation for the Terrestrial Planet Finder Coronagraph. Proc. SPIE. 5899(02) (2005) 1-14.

[5] Y. Y. Ye, Research trends of domestic and international linear motor. National Linear Motor, Modern Drive and System Annual Conference in 2008, Hangzhou, 2008. (In Chinese)

[6] S. Q. Chen and Y. Wang, Experimental Research Isolation Control Based on Active Maglev Isolator. J. Southeast U. 40 (2010) 61-66.(In Chinese) 